\title{
DEVELOPMENT OF AN INTERACTIVE ONLINE ETHICS SCENARIO ACTIVITY FOR ENGINEERING STUDENTS
}

\author{
Peter M. Ostafichuk, Carol P. Jaeger, and Jonathan Nakane \\ Department of Mechanical Engineering, University of British Columbia \\ ostafichuk@mech.ubc.ca
}

\begin{abstract}
This paper describes development and deployment of an online interactive ethical decisionmaking simulation. This tool was piloted in a first-year introduction to engineering course at the University of British Columbia. It used a "choose your own adventure" style of decision-making and narrative to add realism and engagement to what was otherwise viewed by students as dry, uninteresting content. After storyboarding using sticky notes and Visio, the final tool used by students was implemented and deployed using a survey tool (Qualtrics). It featured a scenario with initially incomplete information and the appearance of unethical behaviour by others. It included decision-based branching, but also randomization such that different groups had the story unfold differently, even if they made the same initial decisions. Student feedback on this tool was very positive, suggesting this style of interactive online ethics simulation could be an effective tool for enhancing engagement and learning.
\end{abstract}

Keywords: Ethics, ethical decision-making, simulation, first-year engineering

\section{INTRODUCTION}

Professional and ethical conduct is a common topic covered in first year engineering programs. At the University of British Columbia (UBC), two introduction to engineering courses (APSC 100 and 101) cover this content [1]. There is a two-week module focusing specifically on the profession of engineering, codes of ethics, and ethical decision-making [2]. Our experience with this content is that, while students openly acknowledge the importance of the topics, it is difficult to engage them in active participation. Our most recent course survey had results typical of previous years; across the APSC 100 and 101 courses, the ethics and professionalism module ranked last in terms of student enjoyment, and second (almost tied for first) in terms of importance to engineering.

In an effort to better engage students in the exploration of ethical decision-making, and to continually refresh content, an interactive ethical simulator was developed and piloted in 2019. Our intent was to draw from the benefits of gamification [3], and an engaging narrative. This paper outlines our course context, and then describes the features, development process, and effectiveness of this new tool.

\section{COURSE CONTEXT}

Engineering at UBC has a common first year, with roughly 800-1000 entering students each year. The APSC 100 course is taken in the first term by all entering students and it introduces the topics of the engineering profession, design, decision-making, sustainability, prototyping, CAD, professionalism and ethics. The majority of students continue on to the APSC 101 course in the second term, where they explore topics of design, sustainability, decision-making, and virtual prototyping in greater depth.

The APSC 100 course is delivered in four sequential modules:

- Module 1: introduction to design and stakeholders (3 weeks)

- Module 2: introduction to sustainability and decision-making (4 weeks)

- Module 3: design with an introduction to CAD (3 weeks)

- Module 4: professionalism and ethics (2 weeks)

The activity described in this paper occurs in the last week of Module 4. The key learning goals for Module 4 are the following:

- Describe why professions are regulated

- Describe the risks and advantages of self-regulation

- Describe what Codes of Ethics are and their importance

- Describe the relationship between organizational values, societal values, the law, the Code of Ethics, and personal values

- Explain how personal values, Codes of Ethics, and the law can be used in making defensible decisions in difficult situations

- Apply the UBC Engineering Code of Ethics and/or the Engineers and Geoscientists of British Columbia Code of Ethics to evaluate ethical dilemmas 
- Apply the concept of gradual escalation to identify the correct avenue to report inappropriate behavior

- Describe and give examples of fair, honest, and respectful feedback in the context of peer and instructor evaluations

- Describe the concept of 'best practices'

- Give examples of strategies to mitigate risk in the interpretation of values and ethics

APSC 100 and 101 are delivered in a flipped classroom format, with the majority of content delivered online each week through narrated PowerPoint screencasts, two 50minute large ( $\sim 200$ student) lectures, and one 110-minute small group ( $\sim 50$ student) studio. The online content is completed individually, but the in-class portions (lecture and studio) are heavily team based. The activity described in this paper was delivered as part of the studio session in the last week of Module 4.

\section{DEVELOPMENT AND IMPLEMENTATION}

The new activity introduced this year was designed to add realism to ethical decision-making by providing a branching "choose your own adventure" style narrative to an ethical decision-making scenario. In this activity, choices had consequences, and the full details of the scenario emerged over time, just as in real life. The authors developed the activity through initial brainstorming, mapping and storyboarding using sticky notes on a whiteboard (see Fig. 1).

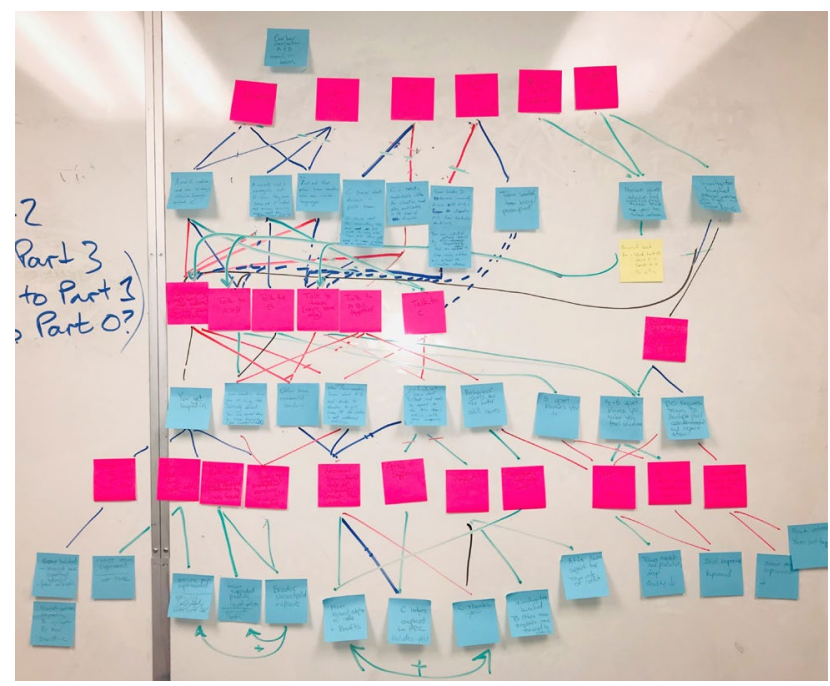

Fig. 1. Sticky note storyboarding

From a single initial scenario starting point, six choices were provided (the top row of dark pink sticky notes in Fig. 1). The next part in the story was randomly determined from up to three branches for each choice, consistent with the choice selected (the second row, consisting of light blue sticky notes in the figure). Here, plausible consequences of the choice were presented, along with richer and more nuanced details for the story. The use of randomization in the branching was done to dissuade teams from attempting to copy choices from other teams, as well as to capture some of the realism and unpredictability in scenarios involving real people. From here there were additional choices, consistent with the evolving story (the middle row of dark pink sticky notes), and the process continued until a "conclusion" to the story was provided after the third choice.

In the scenario, students adopted the persona of a junior member on an engineering design team (i.e., the protagonist of the story). The initial scenario presented to students is shown in Fig. 2, and it consists of the junior member overhearing two senior members use insensitive and inappropriate language.

Fictitious scenario: you are on a student design team and overhear team members $A$ and $B$ using inappropriate language that could be interpreted as degrading or offensive. While you do not believe A and B mean any harm, you imagine their comments could be quite upsetting to team member $C$, in particular, who belongs to the group referred to in the comments. As a first-year student, you have been on the team for only three months, while $A$ and $B$ are senior members with a lot of experience and prominent roles. $C$ is a second-year student, but they too have only been with the team for three months. The team leader, $D$, is a graduate student and has been with the team for over five years now.

University context: UBC Applied Science Professional Development (APSC PD) is a team of people that assists students in many ways, including providing professional and workplace training, facilitating mentoring opportunities, and supporting student design teams. The APSC Dean's Office oversees all engineering activities at UBC; one of these roles is to investigate cases of suspected misconduct, and to act where appropriate. Since 2008, UBC has had a respectful environment statement

(http://www.hr.ubc.ca/respectful-environment/) which among other things, outlines the standards of behaviour expected of all members of the university community and the procedures when those standards are not met.

What do you do?

Fig. 2. Initial scenario description and context. 
The choice to describe the scenario in generic terms and to use "team member A and B" rather than actual names was done such that students could overlay their own interpretations of the characters involved and the nature of the dialog. UBC has 32 extracurricular engineering design teams, with approximately one quarter of engineering undergraduates involved in the teams. There is a strong culture around team participation so this choice of setting for our scenario is one that first year students would identify with easily. While instances of unprofessional behavior of the nature described in the scenario are infrequent, they have occurred and were a source of inspiration for the scenario. Activities such as this can serve to provide an element of formal training for cocurricular activities, and help to reinforce that respectful and ethical behavior is important both in and out of the classroom.

After the initial storyboarding with sticky notes, detailed flowcharting and scripting was done using Visio (see Fig. 3). This figure is included to give a sense of scale, complexity, and overall structure rather than to reveal the full details of each story branch. (For scale, the topmost box in Fig. 3 is the initial scenario description from Fig. 2, and the full diagram is equivalent to a $1200 \mathrm{~mm} \times 800 \mathrm{~mm}$ page with all text in an 8-point font.) As shown in the figure, and to maintain plausibility and consistency in the scenario narrative, with each choice the number of possible outcomes expanded. In total, teams were required to make three choices, which led to a total of 15 distinct possible final outcomes. These ranged from some dissatisfied team members in the scenario with otherwise positive outcomes, to a formal reprimand or discipline of the scenario protagonist, to public backlash through social media and other channels, for example. The outcomes were meant to be plausible, but also to illustrate that actions as well as inactions can have consequences.

The final coding and deployment were done using a survey tool (Qualtrics). This activity was designed for course teams (typically 5 to 6 students) to work through on a single computer during a studio (i.e., tutorial) session. After presenting the scenario of Fig. 2, students were given their first decision choices, reproduced in Fig. 4 and coded as options in a multiple-choice question in the survey tool. These choices were presented in a random order to make each team's experience unique and to remove any perceived pattern in the responses (examining the options of Fig. 4, they escalate from minimal intervention in C1.1 to reporting to authorities in $\mathrm{C} 1.6$ ).

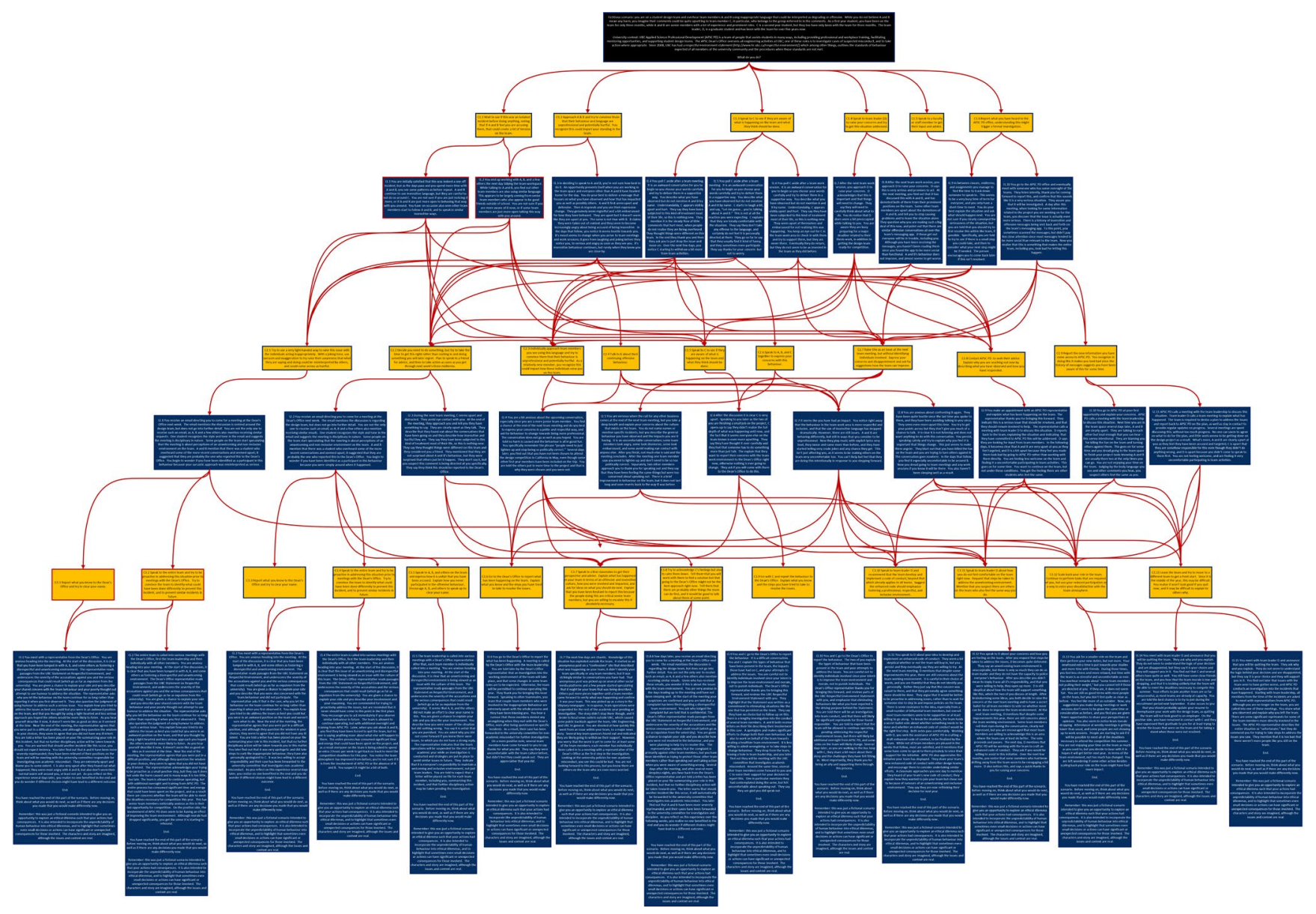

Fig. 3. Overall Visio flowchart

CEEA-ACEG20; Paper 92

Concordia and McGill Universities; June $18-21,2020 \quad-3$ of $5-$ 
C1.1 Wait to see if this was an isolated incident before doing anything, noting that if $A$ and $B$ feel you are accusing them, that could create a lot of tension on the team.

C1.2 Approach $A \& B$ and try to convince them that their behaviour and language are unprofessional and potentially hurtful. You recognize this could impact your standing in the team.

C1.3 Speak to $C$ to see if they are aware of what is happening on the team and what they think should be done.

C1.4 Speak to team leader (D) to raise your concerns and try to get this situation addressed.

C1.5 Speak to a faculty or staff member to get their input and advice.

C1.6 Report what you have heard to the APSC PD office, understanding this might trigger a formal investigation.

Fig. 4. Top-level decision choices. Note that the numbering system (C1.X) was not revealed to students.

Teams discussed and debated the choices provided to them, and then collectively decided on the action they would take. This required teams to draw from course topics (the module learning goals in Section 2), including in particular codes of ethics, ethical theories, ethical decision-making, and other considerations to assist in navigating ethical dilemmas, such as gradual escalation and conflict of interest. Teams were given a textbox in the survey tool to justify their decision before being given the next story element. As depicted in the flowchart of Fig. 3, the next piece of information provided to teams was randomly determined (using the "Randomized Block" feature in Qualtrics), but consistent with the teams' selected choice. This information was displayed along with the next set of multiple-choice options. The remainder of the scenario proceeded in a similar manner.

\section{RESULTS AND DISCUSSION}

The activity was successfully deployed to over 150 teams, with only one minor technical incident affecting one team (due to a coding error on our part). It took most teams 20-30 minutes to complete the full scenario, including discussing choices and justifying their decisions in writing.

Student feedback towards the activity was highly positive. At the conclusion of the activity, several optional survey questions were provided (an advantage of deploying the activity using a survey tool). Teams were asked to rate the activity across several factors using a 0 10 scale, with results shown in Fig. 5:

- Difficulty: How easy was it for your team to reach consensus in making each decision? $(0=$ very difficult, 10 = very easy)

- Plausibility: How plausible/realistic did this scenario seem, including the choices you were given and the consequences of your decisions? $(0=$ very implausible, $10=$ very plausible)

- Discussion: Comparing this ethical simulation activity to a traditional activity (where you describe in writing the steps you would follow in addressing an ethical dilemma), which one do you think promotes richer team discussion? $(0=$ traditional, $10=$ this activity)

- Skill: Which activity do you think is more effective at developing skill in navigating ethical dilemmas? $(0=$ traditional, 10 this activity $)$

- Enjoyment: Which activity do you think is more interesting and/or enjoyable to work through? $(0=$ traditional, $10=$ this activity)
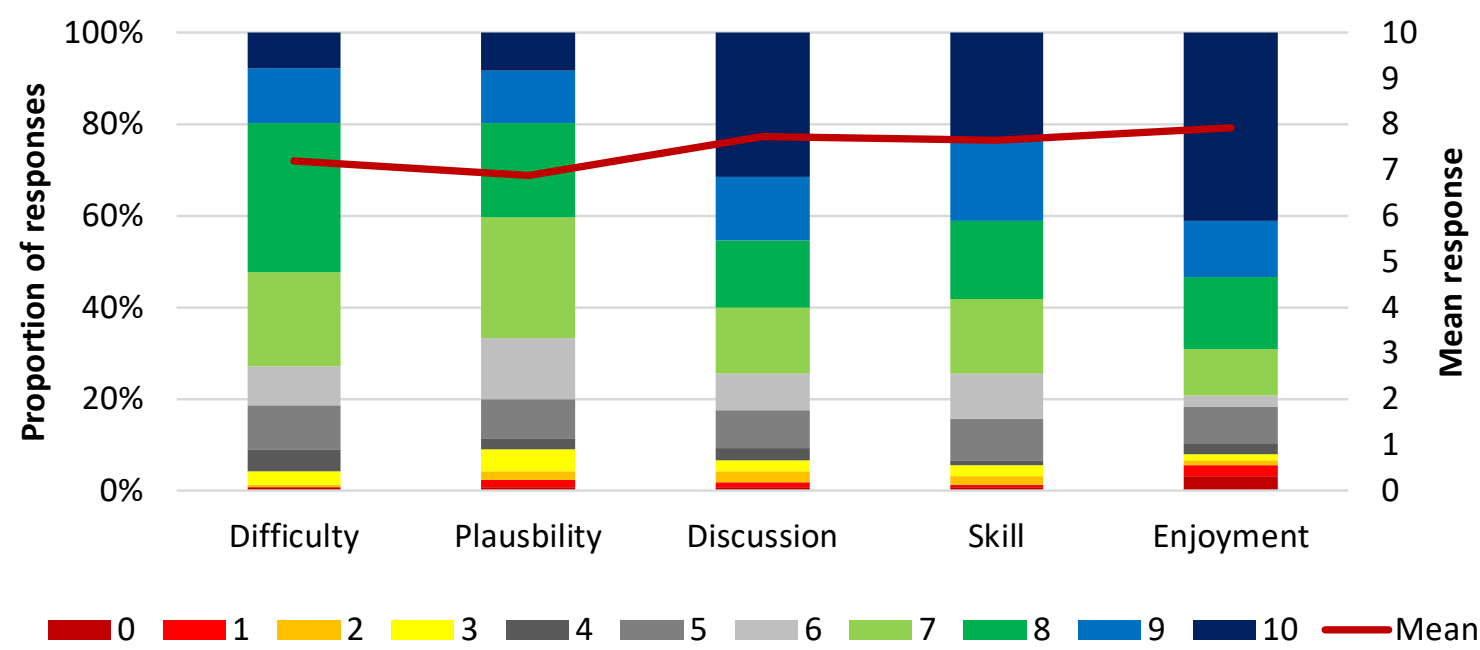

Fig. 5. Activity rating through team exit survey 
The mean ratings provided on the $0-10$ scale reveal broad student support across the different aspects of the activity. Of note, teams gave an average rating of 7.7 for improving intrateam discussion compared to traditional activities ( $82 \%$ positive, $32 \%$ gave 10/10), 7.6 for enhancing skill development in ethical decision-making compared to traditional activities ( $84 \%$ positive, $24 \%$ gave $10 / 10)$, and 7.9 for increasing activity enjoyment $(82 \%$ positive, $41 \%$ gave 10/10). Written feedback, and feedback from tutorial instructors and teaching assistants, was also uniformly positive.

In a separate exit survey for this module, students rated this activity as the most effective of the six main team studio activities (as shown in Fig. 6). This was an optional and anonymous survey, completed individually by students, and thus provides a reassuring complement to the team responses in Fig. 5. In this case, individuals were asked to rate each activity on a four-point scale $(1=$ "not at all effective," 2 = "somewhat effective," 3 = "effective," and $4=$ "very effective"), and this activity received an average rating of 3.1 ("effective").

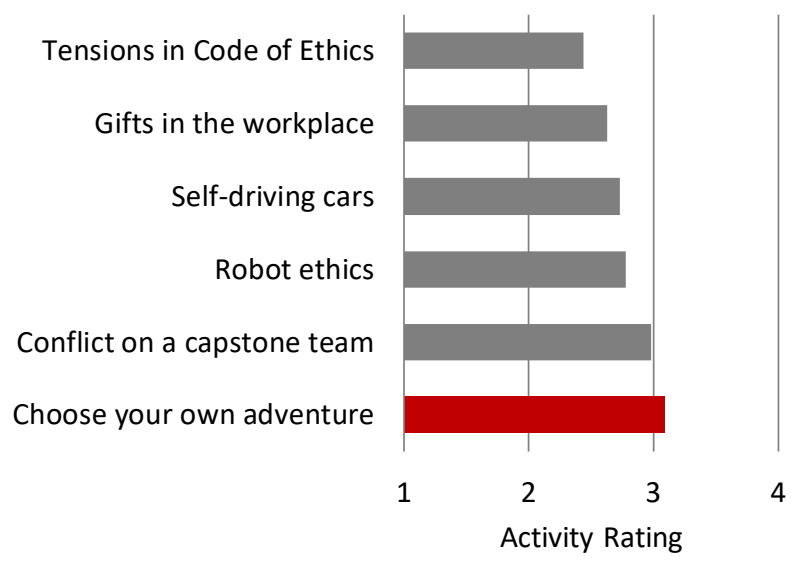

Fig. 6. Activity rating through module exit survey

\section{CONCLUSIONS AND IMPLICATIONS}

Professionalism and ethics are elements of typical first-year engineering programs. While students will indicate these are important topics for engineers, they also report a lack of interest in engaging with them. An interactive online simulation tool was created using a "choose your own adventure" structure in an attempt to increase realism and engagement in our introduction to engineering course at UBC.

We created this tool through storyboarding (with sticky notes on a whiteboard), detailed flow charting (using Visio), and then ultimate implementation using survey software (Qualtrics). The tool was deployed in November 2019 to all 150 teams in the UBC APSC 100 introduction to engineering course.

Overall, the online tool was shown to increase student engagement in ethical decision-making. Importantly, students also reported that it helped to promote discussion within their teams and to enhance the development of ethical decision-making skills. Other feedback collected through course surveys and teaching assistant and instructor observations of students was also very positive. We believe the successes of this activity stemmed from the gamification of an otherwise static or linear learning experience, and the nuances and narrative that emerged as the story in the activity unfolded. As a result of this success, we are currently discussing ways to duplicate this experience in a second professionalism and ethics scenario in our course, or possibly to a separate decision-making topic for a different course module.

\section{REFERENCES}

[1] P. M. Ostafichuk, C. P. Jaeger, J. Nakane, S. Nesbit, N. Ellis and J. Sibley, "Redesigning the UBC First Year Introduction to Engineering: Successes and Challenges," in Proceedings of the Canadian Engineering Education Association (CEEA) Conference, Halifax, 2016.

[2] C. P. Jaeger and P. M. Ostafichuk, "A Framework for Exploring Ethical Dilemmas in a First Year Engineering Course," in Proceedings of the Canadian Engineering Education Association (CEEA) Conference, Toronto, 2017.

[3] J. Hamari, J. Koivisto and H. Sarsa, "Does Gamification Work? -- A Literature Review of Empirical Studies on Gamification," in 47th Hawaii International Conference on System Science, Hawaii, 2014. 